\title{
WEAK-RENORMALIZED SOLUTIONS FOR A SYSTEM THAT MODELS NON-ISOTHERMAL SOLIDIFICATION
}

\author{
ENRIQUE FERNÁNDEZ-CARA*, CRISTINA VAZ ${ }^{\dagger}$ \\ * Departamento EDAN, University of Sevilla, Sevilla, Spain. \\ ${ }^{\dagger}$ Departamento de Matemàtica, Federal University of Parà, PA, Brazil. \\ cara@us.es, cvaz@ufpa.br
}

\begin{abstract}
The aim of this paper is to prove the existence of weak-renormalized solutions to a system of the Navier-Stokes-Boussinesq kind. This system may be regarded as a modified version of the non-isothermal solidification problem with melt convection. This task will be accomplished satisfactorily in the two-dimensional case. Some nontrivial and deep difficulties will be found, however, in three dimensions in space.
\end{abstract}

Key words: Parabolic PDEs, renormalized solutions, solidification models, NavierStokes equations.

AMS subject classifications: $\quad 35 K 60,35 Q 30,76 D 05,80 A 22$

\section{Introduction}

This paper deals with the nonlinear system

$$
\begin{array}{r}
\varphi_{t}-\xi^{2} \Delta \varphi+u \cdot \nabla \varphi=\varphi(\varphi-1)(1-2 \varphi)+\theta \quad \text { in } Q, \\
\theta_{t}-\operatorname{div}(\kappa(\varphi, \theta) \nabla \theta)+u \cdot \nabla \theta=\nu(\varphi, \theta) D(u): D(u) \quad \text { in } Q, \\
u_{t}-\operatorname{div}(\nu(\varphi, \theta) D(u))+(u \cdot \nabla) u+\nabla p=f, \quad \operatorname{div} u=0 \quad \text { in } Q, \\
\varphi=0, \quad \theta=0, \quad u=0 \quad \text { on } \Sigma, \\
\varphi(x, 0)=\varphi_{0}(x), \quad \theta(x, 0)=\theta_{0}(x), \quad u(x, 0)=u_{0}(x) \quad \text { in } \Omega,
\end{array}
$$

where $\Omega \subset \mathbb{R}^{N}$ is an open and bounded domain with a $C^{2}$ boundary $(N=2$ or $N=3), T>0$ is given and $Q=\Omega \times(0, T)$ denotes a space-time cylinder with lateral surface $\Sigma=\partial \Omega \times(0, T)$.

The structure of this system is typical in non-isothermal solidification problems with melt convection $[1,3,10]$; in this particular context, (1) is called the phase-field

Received: August 31, 2011. Accepted: Juny 5, 2012.

The first author has been partially supported by DGES-MICINN, Grant MTM2010-15592. 
equation and is essentially the same found in [10] with an advection term. The other equations are standard and straightforward consequences of the usual physical balance laws (energy, linear momentum and mass).

The unknowns are the phase-field function $\varphi$, the temperature $\theta$, the velocity field $u$ and the hydrostatic pressure $p$; $\xi$ is a positive constant related to the width of the transitions layers; $\kappa$ and $\nu$ are strictly positive functions that depend on $\varphi$ and $\theta$ and must be viewed as a heat diffusion and a kinematic fluid viscosity, respectively; $f$ is an external field; $D(u)$ is the deformation tensor, i.e.

$$
D(u)=\frac{1}{2}\left(\nabla u+\nabla u^{T}\right)
$$

and $\varphi_{0}, \theta_{0}$ and $u_{0}$ are given functions.

Throughout this paper, we will denote by $C$ or $M$ generic constants depending only on known quantities, which will be indicated frequently.

A great deal of attention has been paid to phase-field models for solidification processes during the last two decades by several authors; see for example [10, 11, $20,3,1]$. In these works, many situations and many different hypotheses have been considered, in special the possibility of motion of molten material during solidification processes. In our case, the molten material is assumed to behave as an incompressible fluid with variable viscosity. The resulting system can thus be viewed as a generalization of the models considered in the previous papers.

We will consider the (simplified) case where the latent heat in the energy equation is very small and can be neglected. Notice that the equation (2) needs a special treatment due to the nonlinear right-hand side, that only belongs to $L^{1}(Q)$ since, in general, $D(u)$ only belongs to $L^{2}(Q)^{N \times N}$. For this reason, we will consider the notion of renormalized solutions adapted to our setting.

Renormalized solutions to PDEs were first introduced by DiPerna and P.L. Lions $[13,12]$ in the context of Boltzmann-like equations. Later, they have also been considered in other situations; let us mention in particular the contributions of Blanchard, Boccardo, Murat and their co-workers in the framework of second-order elliptic and parabolic PDEs; see [6, 7, 4, 5] and the references therein; see also [19] and [8] for more related results.

In order to solve (1)-(5), we will use regularization techniques, truncations, appropriate estimates and the compactness of approximate solutions.

This paper is organized as follows.

In Section 2, we fix the notation and we introduce some functional spaces. We also recall certain interpolation and embedding results. We enumerate the hypotheses, we introduce the concept of weak-renormalized solution adapted to our context and we state the main result of the paper.

In Section 3, we investigate the solvability of some auxiliary problems.

Section 4 is devoted to present the proof of the existence result for two-dimensional flows; it is split in three steps, namely, the formulation and resolution of regularized problems, the obtention of estimates, and the passage to the limit. 


\section{Preliminaries}

\subsection{Notation and spaces}

For any $q \geq 1$, we denote by $L^{q}(\Omega)$ the standard Lebesgue space with usual norm denoted by $\|\cdot\|_{q, \Omega}$. For any nonnegative integer $m, W^{m, q}(\Omega)$ is the standard Sobolev space with usual norm denoted by $\|\cdot\|_{m, q, \Omega}$. The space $W_{0}^{m, q}(\Omega)$ is the closure with respect to the norm $\|\cdot\|_{m, q, \Omega}$ of the space $C_{0}^{\infty}(\Omega)$ of $C^{\infty}$ functions with compact support in $\Omega$. We refer for instance to [14] for more details on the previous spaces.

The following result from [21] will be used below:

$$
\iint_{Q}|v|^{\tau} d x d t \leq C\|v\|_{L^{\infty}\left(0, T ; L^{p}(\Omega)\right)}^{p q / N} \iint_{Q}|\nabla v|^{q} d x d t
$$

for every $v \in L^{q}\left(0, T ; W_{0}^{1, q}(\Omega)\right) \cap L^{\infty}\left(0, T ; L^{p}(\Omega)\right)$ with $p, q \geq 1$ and $\tau=$ $q(N+p) / N$.

For the analysis of the motion equation (3), we will need other function spaces. Thus, let us set $\mathcal{V}=\left\{v \in C_{0}^{\infty}(\Omega)^{N}: \operatorname{div} v=0\right\}$; we will denote the closures of $\mathcal{V}$ in $L^{2}(\Omega)^{N}$ and $H_{0}^{1}(\Omega)^{N}$ respectively by $H$ and $V$. Then, $H$ and $V$ are Hilbert spaces for the corresponding norms and one has

$$
H=\left\{v \in L^{2}(\Omega)^{N}: \operatorname{div} v=0 \text { in } \Omega, v \cdot n=0 \text { on } \partial \Omega\right\}
$$

and

$$
V=\left\{v \in H_{0}^{1}(\Omega)^{N}: \operatorname{div} v=0 \text { in } \Omega\right\} .
$$

The general properties of these spaces can be found for instance in [22].

In the sequel, we will use the following truncation function: for any positive real number $R$, we set

$$
T_{R}(s)=s \text { if }|s| \leq R \text { and } T_{R}(s)=R \operatorname{sign}(s) \text { if }|s|>R,
$$

where $\operatorname{sign}(s)=0$ if $s=0$ and $\operatorname{sign}(s)=s /|s|$ if $s \neq 0$.

Since $T_{R}$ is a Lipschitz function, for any function $v \in W_{0}^{1, q}(\Omega)$ one has $T_{R}(v) \in$ $W_{0}^{1, q}(\Omega)$ and the chain rule for the differentiation of $T_{R}(v)$ holds true, that is,

$$
\nabla T_{R}(v)=T_{R}^{\prime}(v) \nabla v \text { a.e. in } \Omega .
$$

We will also have to consider the following set:

$$
\begin{gathered}
\mathcal{L}(0, T, \Omega)=\left\{v \in L^{\infty}\left(0, T ; L^{1}(\Omega)\right): T_{R}(v) \in L^{2}\left(0, T ; H_{0}^{1}(\Omega)\right) \forall R>0,\right. \\
\left.\lim _{n \rightarrow+\infty} \frac{1}{n} \int_{A_{n}(v)}|\nabla v|^{2} d x d t=0\right\} .
\end{gathered}
$$

Here and in the sequel, $A_{n}(v)$ stands for the set

$$
A_{n}(v)=\{(x, t) \in Q: n \leq|v(x, t)| \leq 2 n\} .
$$

We will make use of the following lemma, due to Boccardo and Gallouët (see [7]; see also [18]): 
Lemma 1 Assume that $v \in L^{\infty}\left(0, T ; L^{1}(\Omega)\right), T_{R}(v) \in L^{2}\left(0, T ; H_{0}^{1}(\Omega)\right)$ for all $R>0$ and there exists $M>0$ such that

$$
\|v\|_{L^{\infty}\left(0, T ; L^{1}(\Omega)\right)} \leq M \text { and } \iint_{Q}\left|\nabla T_{R}(v)\right|^{2} d x d t \leq M R \quad \forall R>0 .
$$

Then, for all $1<q<(N+2) /(N+1)$, one has

$$
v \in L^{q}\left(0, T ; W_{0}^{1, q}(\Omega)\right) \text { and }\|v\|_{L^{q}\left(0, T ; W_{0}^{1, q}(\Omega)\right)} \leq C(q) M .
$$

\subsection{Hypotheses and main result}

Along this work, we will assume that the following hypotheses hold:

(H) $\quad\left\{\begin{array}{l}f \in L^{2}(Q)^{N}, \varphi_{0} \in L^{2}(\Omega), u_{0} \in H, \theta_{0} \in L^{1}(\Omega), \\ \nu, \kappa \in C^{0}(\mathbb{R} \times \mathbb{R}), 0<\nu_{1} \leq \nu \leq \nu_{2} \text { and } 0<\kappa_{1} \leq \kappa \leq \kappa_{2} .\end{array}\right.$

We introduce now the definition of weak-renormalized solution to (1)-(5):

Definition 1 It will be said that $(\varphi, \theta, u)$ is a (weak-renormalized) solution to (1)-(5) if the following conditions are satisfied:

1. $u \in L^{\infty}(0, T ; H) \cap L^{2}(0, T ; V), \varphi \in L^{\infty}\left(0, T ; L^{2}(\Omega)\right) \cap L^{2}\left(0, T ; H_{0}^{1}(\Omega)\right) \cap$ $L^{4}(Q)$ and $\theta \in \mathcal{L}(0, T, \Omega)$.

2. $\varphi$ solves (1) in the usual weak sense and $\left.\varphi\right|_{t=0}=\varphi_{0}$.

3. $u$ solves (3) in the usual weak sense (together with some $p \in \mathcal{D}^{\prime}(Q)$ ) and $\left.u\right|_{t=0}=u_{0}$.

4. For any $\beta \in W^{2, \infty}(\mathbb{R})$ such that $\operatorname{Supp} \beta^{\prime}$ is compact and for any $\eta \in$ $C^{1}\left([0, T] ; H_{0}^{1}(\Omega)\right) \cap L^{\infty}(Q)$ such that $\left.\eta\right|_{t=T}=0$, we have

$$
\begin{gathered}
-\iint_{Q} \beta(\theta) \eta_{t} d x d t+\iint_{Q} \kappa(\varphi, \theta) \nabla \beta(\theta) \cdot \nabla \eta d x d t \\
+\iint_{Q} \kappa(\varphi, \theta) \nabla \theta \cdot \nabla \beta^{\prime}(\theta) \eta d x d t-\iint_{Q}\left(u \cdot \nabla \beta^{\prime}(\theta)\right) \eta d x d t \\
=\iint_{Q} \beta^{\prime}(\theta) \nu(\varphi, \theta) D(u): D(u) \eta d x d t+\int_{\Omega} \beta\left(\theta_{0}\right) \eta(x, 0) d x .
\end{gathered}
$$

We can now state our main result in this paper:

Theorem 2 Assume that $N=2$ and (H) holds. Then, there exists at least one solution to (1)-(5). 


\section{Some auxiliary problems}

In order to prove theorem 2, it is convenient to first consider and solve some auxiliary problems.

Let $\left\{\rho_{\epsilon}\right\}$ be a regularizing sequence in $\mathbb{R}^{N}$. For any $\epsilon>0$ and any $v \in H$, we will denote by $R_{\epsilon} v$ the following function:

$$
R_{\epsilon} v:=\rho_{\epsilon} * \tilde{v} .
$$

Here, $\tilde{v}$ is the extension by zero of $v$ to the whole $\mathbb{R}^{N}$.

Recall that $R_{\epsilon} v \in C^{\infty}\left(\mathbb{R}^{N}\right)^{N}, \nabla \cdot\left(R_{\epsilon} v\right)=0$ in $\Omega$ and we have in particular

$$
\left\|R_{\epsilon} v\right\|_{m, q, \Omega} \leq C(m, p, \epsilon)\|v\|_{2, \Omega}
$$

for all $m$ and $q$.

The first auxiliary problem is the following:

$$
\begin{array}{r}
u_{t}-\operatorname{div}(m(x, t) D(u))+\left(\left(R_{\epsilon} u\right) \cdot \nabla\right) u+\nabla p=f, \quad \operatorname{div} u=0 \quad \text { in } Q, \\
u=0 \quad \text { on } \Sigma, \\
u(x, 0)=u_{0}(x) \quad \text { in } \Omega .
\end{array}
$$

Here, we assume that

$$
\left\{\begin{array}{l}
f \in L^{2}(Q)^{N}, \quad u_{0} \in H \\
m \in L^{\infty}(Q), \quad 0<\nu_{1} \leq m(x, t) \leq \nu_{2} \text { a.e. }
\end{array}\right.
$$

The existence and uniqueness of a weak solution to (8)-(10) can be proved via a Galerkin method for instance like in [17] or [22] for the classical Navier-Stokes equations. In that way, the following is obtained:

Proposition 3 Let the assumptions (11) be satisfied. Then there exists exactly one solution to (8)-(10), with

$$
u \in L^{2}(0, T ; V) \cap C^{0}([0, T] ; H), \quad u_{t} \in L^{2}\left(0, T ; V^{\prime}\right) .
$$

Furthermore, one has

$$
\left\{\begin{array}{l}
\|u\|_{L^{2}(0, T ; V)}+\|u\|_{L^{\infty}(0, T ; H)}+\left\|u_{t}\right\|_{L^{\sigma}\left(0, T ; V^{\prime}\right)} \leq C, \\
\left\|u_{t}\right\|_{L^{2}\left(0, T ; V^{\prime}\right)} \leq C(\epsilon)
\end{array}\right.
$$

where $\sigma=2$ if $N=2$ and $\sigma=4 / 3$ if $N=3$ and $C$ (resp. $C(\epsilon)$ ) depends on $\Omega, T$, $\|f\|_{L^{2}(Q)},\left\|u_{0}\right\|_{H}, \nu_{1}$ and $\nu_{2}$ (resp. these data and $\epsilon$ ).

Next, we consider a second auxiliary problem, closely related to the phase-field equation in our original system:

$$
\begin{array}{r}
\varphi_{t}-\xi^{2} \Delta \varphi+u \cdot \nabla \varphi=\varphi(\varphi-1)(1-2 \varphi)+h \quad \text { in } Q \\
\varphi=0 \quad \text { on } \Sigma, \\
\varphi(x, 0)=\varphi_{0}(x) \quad \text { in } \Omega,
\end{array}
$$


where

$$
h \in L^{1}\left(0, T ; L^{2}(\Omega)\right), u \in L^{2}(0, T ; V) \cap L^{\infty}(0, T ; H), \varphi_{0} \in L^{2}(\Omega) .
$$

The following result can also be proved by a Galerkin-compactness method.

Proposition 4 Let the assumptions (15) be satisfied. Then, there exists a unique solution to (12)-(14), with

$$
\left\{\begin{array}{l}
\varphi \in L^{2}\left(0, T ; H_{0}^{1}(\Omega)\right) \cap C_{w}^{0}\left([0, T] ; L^{2}(\Omega)\right) \cap L^{4}(Q), \\
\varphi_{t} \in L^{1}\left(0, T ; L^{2}(\Omega)\right)+L^{\sigma}\left(0, T ; H^{-1}(\Omega)\right)
\end{array}\right.
$$

and the norms in these spaces bounded by a constant that only depends on $\Omega, T$, $\|h\|_{L^{1}\left(0, T ; L^{2}(\Omega)\right)},\|u\|_{L^{2}(0, T ; V)}+\|u\|_{L^{\infty}(0, T ; H)}$ and $\left\|\varphi_{0}\right\|_{L^{2}}$. If $N=2$, one also has $\varphi \in C^{0}\left([0, T] ; L^{2}(\Omega)\right)$.

Sketch of the proof: Let us first explain how the existence of $\varphi$ can be established.

Let us denote by $\varphi^{m}:\left[0, T_{m}\right) \mapsto H_{0}^{1}(\Omega)$ the approximations that can be obtained from a standard Galerkin scheme where the basis functions are the eigenfunctions of the Dirichlet Laplacian in $\Omega$. In principle, $\varphi^{m}$ is only locally defined, i.e. we can have $T_{m}<T$.

By setting $H(z):=z(z-1)(2 z-1) \equiv 2 z^{3}-3 z^{2}+z$, it is clear that

$$
\frac{1}{2} \frac{d}{d t}\left\|\varphi^{m}\right\|_{2, \Omega}^{2}+\xi^{2}\left\|\nabla \varphi^{m}\right\|_{2, \Omega}^{2}+\int_{\Omega} H\left(\varphi^{m}\right) \varphi^{m} d x=\left(h, \varphi^{m}\right)_{2, \Omega}
$$

for all $0 \leq t<T_{m}$. Since $H(z) z \equiv 2 z^{4}-3 z^{3}+z^{2}$, we have $H(z) z \geq z^{4}-C$ for all $z$. After integration in time, we get the following in $\left[0, T_{m}\right)$ :

$$
\begin{aligned}
& \left\|\varphi^{m}(t)\right\|_{2, \Omega}^{2}+\xi^{2} \int_{0}^{t}\left\|\nabla \varphi^{m}(s)\right\|_{2, \Omega}^{2} d s+\int_{0}^{t}\left\|\varphi^{m}(s)\right\|_{4, \Omega}^{4} d s \\
& \quad \leq\left\|\varphi_{0}\right\|_{2, \Omega}^{2}+\frac{1}{2}\|h\|_{L^{1}\left(0, T ; L^{2}(\Omega)\right)}^{2}+\frac{1}{2} \sup _{\left[0, T_{m}\right)}\left\|\varphi^{m}(s)\right\|_{2, \Omega}^{2}+C,
\end{aligned}
$$

where $C$ only depends on $\Omega$ and $T$. Consequently, $T_{m}=T$, the $\varphi^{m}$ are globally defined and, furthermore,

$$
\varphi^{m} \in \text { bounded set in } L^{2}\left(0, T ; H_{0}^{1}(\Omega)\right) \cap L^{\infty}\left(0, T ; L^{2}(\Omega)\right) \cap L^{4}(Q) .
$$

On the other hand, since $\varphi_{t}^{m}(t)$ can be written as the orthogonal projection of $\left(\xi^{2} \Delta \varphi^{m}-u \cdot \nabla \varphi^{m}-H\left(\varphi^{m}\right)+h\right)(t)$ on the space spanned by the first $m$ eigenfunctions, one has

$$
\begin{aligned}
\left\|\varphi_{t}^{m}\right\|_{-1,2, \Omega} & \leq\left\|\xi^{2} \Delta \varphi^{m}-u \cdot \nabla \varphi^{m}-H\left(\varphi^{m}\right)+h\right\|_{-1,2, \Omega} \\
& \leq C\left(\|\nabla \varphi\|_{2, \Omega}+\left\|u \cdot \nabla \varphi^{m}\right\|_{-1,2, \Omega}+\|\varphi\|_{4, \Omega}^{3}+\|h\|_{2, \Omega}\right)
\end{aligned}
$$

in $(0, T)$, whence it is easy to deduce that

$$
\varphi_{t}^{m}-h \in \text { bounded set in } L^{\sigma}\left(0, T ; H^{-1}(\Omega)\right) .
$$


From (17) and (18), using standard arguments, we can extract a sequence that converges to a solution to (12)-(14) and satisfies (16).

The uniqueness of $\varphi$ can be obtained by applying Gronwall's lemma.

More precisely, let us assume that (for instance) $N=3$, let $\varphi^{1}$ and $\varphi^{2}$ be two solutions to (12)-(14) satisfying (16) and let us set $\varphi:=\varphi^{1}-\varphi^{2}$. Notice that

$$
\begin{aligned}
\left(H\left(z_{1}\right)-H\left(z_{2}\right)\right. & )\left(z_{1}-z_{2}\right) \\
& =2\left(z_{1}^{3}-z_{2}^{3}\right)\left(z_{1}-z_{2}\right)-3\left(z_{1}^{2}-z_{2}^{2}\right)\left(z_{1}-z_{2}\right)+\left(z_{1}-z_{2}\right) \\
\geq & -3\left(z_{1}^{2}-z_{2}^{2}\right)\left(z_{1}-z_{2}\right) \\
& =-3\left(z_{1}+z_{2}\right)\left(z_{1}-z_{2}\right)^{2}
\end{aligned}
$$

for all $z_{1}, z_{2} \in \mathbb{R}$. Then, one has:

$$
\begin{aligned}
\frac{1}{2} \frac{d}{d t} & \|\varphi\|_{2, \Omega}^{2}+\xi^{2}\|\nabla \varphi\|_{2, \Omega}^{2}=-\int_{\Omega}\left(H\left(\varphi^{1}\right)-H\left(\varphi^{2}\right)\right) \varphi d x \\
& \leq 3 \int_{\Omega}\left(\left|\varphi^{1}\right|+\left|\varphi^{2}\right|\right)|\varphi|^{2} d x \\
& \leq C\left(\left\|\varphi^{1}\right\|_{3, \Omega}+\left\|\varphi^{2}\right\|_{3, \Omega}\right)\|\varphi\|_{2, \Omega}\|\varphi\|_{6, \Omega} \\
& \leq C\left(\left\|\varphi^{1}\right\|_{3, \Omega}^{2}+\left\|\varphi^{2}\right\|_{3, \Omega}^{2}\right)\|\varphi\|_{2, \Omega}^{2}+\frac{\xi^{2}}{2}\|\nabla \varphi\|_{2, \Omega}^{2}
\end{aligned}
$$

in $(0, T)$. Since $\left\|\varphi^{i}\right\|_{3, \Omega}^{2} \leq C\left\|\varphi^{i}\right\|_{2, \Omega}\left\|\nabla \varphi^{i}\right\|_{2, \Omega}$, we see that, for some $F \in L^{2}(0, T)$, one has

$$
\frac{d}{d t}\left\|\varphi^{i}\right\|_{2, \Omega}^{2}+\xi^{2}\left\|\nabla \varphi^{i}\right\|_{2, \Omega}^{2} \leq F(t)\left\|\varphi^{i}\right\|_{2, \Omega}^{2}
$$

These inequalities, together with Gronwall's lemma, imply $\varphi \equiv 0$, whence we necessarily have $\varphi^{1} \equiv \varphi^{2}$.

\section{Proof of theorem 2}

\subsection{An auxiliary regularized problem}

We begin by introducing some notation. Thus, for any $\epsilon>0$, we set:

(i) $\theta_{0 \epsilon}=T_{1 / \epsilon}\left(\theta_{0}\right)$.

(ii) $g_{\epsilon}=T_{1 / \epsilon}\left(\nu\left(\varphi_{\epsilon}, \theta_{\epsilon}\right) D\left(u_{\epsilon}\right): D\left(u_{\epsilon}\right)\right)$.

We will begin the proof with no restriction on the dimension $(N=2$ or $N=3)$. We consider the following regularized version of (1)-(5):

$$
\begin{aligned}
\varphi_{\epsilon, t}-\xi^{2} \Delta \varphi_{\epsilon}+u_{\epsilon} \cdot \nabla \varphi_{\epsilon}=\varphi_{\epsilon}\left(\varphi_{\epsilon}-1\right)\left(1-2 \varphi_{\epsilon}\right)+\theta_{\epsilon} & \text { in } Q, \\
\varphi_{\epsilon}=0 \text { on } \Sigma, \quad \varphi_{\epsilon}(x, 0)=\varphi_{0}(x) & \text { in } \Omega, \\
\theta_{\epsilon, t}-\operatorname{div}\left(\kappa\left(\varphi_{\epsilon}, \theta_{\epsilon}\right) \nabla \theta_{\epsilon}\right)+u_{\epsilon} \cdot \nabla \theta_{\epsilon}=g_{\epsilon} & \text { in } Q, \\
\theta_{\epsilon}=0 \quad \text { on } \Sigma, \quad \theta_{\epsilon}(x, 0)=\theta_{0 \epsilon}(x) & \text { in } \Omega, \\
u_{\epsilon, t}-\operatorname{div}\left(\nu\left(\varphi_{\epsilon}, \theta_{\epsilon}\right) D\left(u_{\epsilon}\right)\right)+\left(\left(R_{\epsilon} u_{\epsilon}\right) \cdot \nabla\right) u_{\epsilon}+\nabla p_{\epsilon}=f, \operatorname{div} u_{\epsilon}=0 & \text { in } Q, \\
u_{\epsilon}=0 \quad \text { on } \Sigma, \quad u_{\epsilon}(x, 0)=u_{0}(x) & \text { in } \Omega .
\end{aligned}
$$


We then have the following existence result:

Proposition 5 Let the assumptions $(\mathbf{H})$ be fulfilled. Then, for each $\epsilon>0$, there exists at least one solution $\left(\varphi_{\epsilon}, \theta_{\epsilon}, u_{\epsilon}\right)$ to (19)-(24), with

$$
\left\{\begin{array}{l}
\varphi_{\epsilon}, \theta_{\epsilon} \in L^{2}\left(0, T ; H_{0}^{1}(\Omega)\right) \cap C_{w}^{0}\left([0, T] ; L^{2}(\Omega)\right) \\
u_{\epsilon} \in L^{2}(0, T ; V) \cap C_{w}^{0}([0, T] ; H)
\end{array}\right.
$$

Proof: The proof can be obtained from a standard application of Schauder's or LeraySchauder's fixed point theorem.

Let us consider the mapping $\Lambda_{\epsilon}$ that associates to each $(\varphi, \theta) \in L^{1}(Q)^{2}$, first, the unique solution $u_{\epsilon}$ to (23)-(24) with $\nu\left(\varphi_{\epsilon}, \theta_{\epsilon}\right)$ replaced by $\nu(\varphi, \theta)$; then, the unique solution $\theta_{\epsilon}$ to (21)-(22) with $g_{\epsilon}$ replaced by $T_{1 / \epsilon}\left(\nu(\varphi, \theta) D\left(u_{\epsilon}\right): D\left(u_{\epsilon}\right)\right)$ and $\kappa\left(\varphi_{\epsilon}, \theta_{\epsilon}\right)$ replaced by $\kappa(\varphi, \theta)$; finally, the unique solution $\varphi_{\epsilon}$ to (19)-(20).

In view of the results in Sections 2 and 3, $\Lambda_{\epsilon}: L^{1}(Q)^{2} \mapsto L^{1}(Q)^{2}$ is well-defined. Furthermore, it is continuous. Indeed, let $(\varphi, \theta)$ and the $\left(\varphi^{n}, \theta^{n}\right)$ be given in $L^{1}(Q)^{2}$, let us set

$$
(\bar{\varphi}, \bar{\theta})=\Lambda_{\epsilon}(\varphi, \theta), \quad\left(\bar{\varphi}^{n}, \bar{\theta}^{n}\right)=\Lambda_{\epsilon}\left(\varphi^{n}, \theta^{n}\right)
$$

and let us assume that $\left(\varphi^{n}, \theta^{n}\right) \rightarrow(\varphi, \theta)$ strongly in $L^{1}(Q)^{2}$. Then

1. $\nu\left(\varphi^{n}, \theta^{n}\right) \rightarrow \nu(\varphi, \theta)$ strongly in all the spaces $L^{p}(Q)^{2}$ with $1 \leq p<+\infty$,

2. The associated $u^{n}$ converge strongly in $L^{2}(0, T ; V)$,

3. $\bar{\theta}^{n} \rightarrow \bar{\theta}$ and $\bar{\theta}_{t}^{n} \rightarrow \bar{\theta}_{t}$ resp. strongly in $L^{2}\left(0, T ; H_{0}^{1}(\Omega)\right)$ and strongly in $L^{2}\left(0, T ; H^{-1}(\Omega)\right)$ and

4. Finally, $\bar{\varphi}^{n} \rightarrow \bar{\varphi}$ and $\bar{\varphi}_{t}^{n} \rightarrow \bar{\varphi}_{t}$ resp. strongly in $L^{2}\left(0, T ; H_{0}^{1}(\Omega)\right)$ and strongly in $L^{2}\left(0, T ; H^{-1}(\Omega)\right)$.

The first of these assertions is evident. The third and the fourth assertions are consequences of the usual energy estimates for parabolic equations. The second one can be justified as follows.

First, from the estimates in proposition 3 , it is clear that $u^{n}$ converges weakly in $L^{2}(0, T ; V)$ to $u$, the solution associated to $(\varphi, \theta)$. Secondly, taking into account that $u_{t}$ and the $u_{t}^{n}$ belong to $L^{2}\left(0, T ; V^{\prime}\right)$, we find the energy identities

$$
\frac{1}{2}\|u(T)\|_{2, \Omega}^{2}+\iint_{Q} \nu(\varphi, \theta)|D(u)|^{2} d x d t=\iint_{Q} f \cdot u d x d t+\frac{1}{2}\left\|u_{0}\right\|_{2, \Omega}^{2}
$$

and

$$
\frac{1}{2}\left\|u^{n}(T)\right\|_{2, \Omega}^{2}+\iint_{Q} \nu\left(\varphi^{n}, \theta^{n}\right)\left|D\left(u^{n}\right)\right|^{2} d x d t=\iint_{Q} f \cdot u^{n} d x d t+\frac{1}{2}\left\|u_{0}\right\|_{2, \Omega}^{2}
$$

for all $n \geq 1$. Consequently,

$$
\begin{gathered}
\lim _{n \rightarrow+\infty}\left[\frac{1}{2}\left\|u^{n}(T)\right\|_{2, \Omega}^{2}+\iint_{Q} \nu\left(\varphi^{n}, \theta^{n}\right)\left|D\left(u^{n}\right)\right|^{2} d x d t\right] \\
=\frac{1}{2}\|u(T)\|_{2, \Omega}^{2}+\iint_{Q} \nu(\varphi, \theta)|D(u)|^{2} d x d t .
\end{gathered}
$$


But this yields the strong convergence of $\left(u^{n}(T), \nu\left(\varphi^{n}, \theta^{n}\right)^{1 / 2} D\left(u^{n}\right)\right)$ in the product space $H \times L^{2}(Q)^{N \times N}$. Since $\nu\left(\varphi^{n}, \theta^{n}\right)$ converges a.e. and is uniformly bounded from above and from below, we deduce that $D\left(u^{n}\right)$ also converges strongly in $L^{2}(Q)^{N \times N}$. In view of Korn's inequality, this is equivalent to the strong convergence of $\nabla u^{n}$ in the same space, that is, the strong convergence of $u^{n}$ in $L^{2}(0, T ; V)$.

Notice that $\Lambda_{\epsilon}$ maps the whole space $L^{1}(Q)^{2}$ into a compact set.

Indeed, let us set

$$
W=\left\{\phi \in L^{2}\left(0, T ; H_{0}^{1}(\Omega)\right): \phi_{t} \in L^{2}\left(0, T ; H^{-1}(\Omega)\right)\right\} .
$$

Recall that, endowed with its natural norm, $W$ is a Hilbert space such that the embedding $W \hookrightarrow L^{1}(Q)$ is compact. Let $(\varphi, \theta)$ be given in $L^{1}(Q)^{2}$ and let us set $(\bar{\varphi}, \bar{\theta})=\Lambda_{\epsilon}(\varphi, \theta)$. Then, the assumptions on $\nu$ and $\kappa$ in $(\mathbf{H})$ and the fact that $\epsilon$ is fixed yield uniform bounds of the norms of $\bar{\varphi}$ and $\bar{\theta}$ in $W$. But this means that $(\bar{\varphi}, \bar{\theta})$ belongs to a fixed compact set of $L^{1}(Q)^{2}$.

Consequently, we can apply Schauder's theorem to $\Lambda_{\epsilon}$ and deduce that this mapping possesses at least one fixed point.

This provides a solution to (19)-(24) and ends the proof.

\subsection{Some a priori estimates}

We will now deduce some a priori estimates for the solutions to (19)-(24), uniform with respect to $\epsilon$.

To this end, we start by applying proposition 3 to (23)-(24) and we obtain:

$$
\left\|u_{\epsilon}\right\|_{L^{2}(0, T ; V)}+\left\|u_{\epsilon}\right\|_{L^{\infty}(0, T ; H)}+\left\|u_{\epsilon, t}\right\|_{L^{\sigma}\left(0, T ; V^{\prime}\right)} \leq C \text {. }
$$

A first consequence is that the $g_{\epsilon}=T_{1 / \epsilon}\left(\nu\left(\varphi_{\epsilon}, \theta_{\epsilon}\right) D\left(u_{\epsilon}\right): D\left(u_{\epsilon}\right)\right)$ are uniformly bounded in $L^{1}(Q)$.

In view of the results in [2], the following estimates hold for $\theta_{\epsilon}$ :

$$
\left\{\begin{array}{l}
\theta_{\epsilon} \in \text { bounded set in } L^{\infty}\left(0, T ; L^{1}(\Omega)\right) \cap L^{1}\left(0, T ; L^{p}(\Omega)\right) \\
\text { for all } 1<p<+\infty \text { if } N=2 \text { and for all } 1<p<3 \text { if } N=3 .
\end{array}\right.
$$

Furthermore, arguing as in [6], we see that there exists $M$ such that

$$
\iint_{Q}\left|\nabla T_{R}\left(\theta_{\epsilon}\right)\right|^{2} d x d t \leq M R \text { and } \frac{1}{n} \iint_{\left\{n \leq\left|\theta_{\epsilon}\right| \leq 2 n\right\}}\left|\nabla \theta_{\epsilon}\right|^{2} d x d t \leq M
$$

for all $R>0$ and $n \geq 1$.

From lemma 1, we get:

$$
\theta_{\epsilon} \in \text { bounded set in } L^{q}\left(0, T ; W_{0}^{1, q}(\Omega)\right) \forall 1<q<\frac{N+2}{N+1} .
$$

Combining (26), (28) and the embedding result (6), we deduce that

$$
\theta_{\epsilon} \in \text { bounded set in } L^{\tau}(Q) \forall 1<\tau<\frac{N+2}{N} .
$$


On the other hand, from the PDE satisfied by $\theta_{\epsilon}$, the fact that $g_{\epsilon}$ is uniformly bounded in $L^{1}(Q)$, (25) and (26), one has:

$$
\theta_{\epsilon, t} \in \text { bounded set in } L^{1}\left(0, T ; W^{-1, a}(\Omega)\right) \forall 1<a<\bar{a},
$$

where $\bar{a}=4 / 3$ if $N=2$ and $\bar{a}=6 / 5$ if $N=3$.

Indeed, from the usual interpolation results, it is clear from (26) that

$$
\theta_{\epsilon} \in \text { bounded set in } L^{r}\left(0, T ; L^{s}(\Omega)\right) \forall 1<r<+\infty, \forall 1<s<\bar{s}(r) \text {, }
$$

where $\bar{s}(r)=r /(r-1)$ if $N=2$ and $\bar{s}(r)=3 r /(3 r-2)$ if $N=3$. On the other hand, (25) implies

$$
u_{\epsilon} \in \text { bounded set in } L^{\rho}\left(0, T ; L^{\sigma}(\Omega)\right) \forall 2<\rho<+\infty, \forall 1<\sigma<\bar{\sigma}(\rho),
$$

where $\bar{\sigma}(\rho)=2 \rho /(\rho-2)$ if $N=2$ and $\bar{\sigma}(\rho)=6 \rho /(3 \rho-4)$ if $N=3$. Consequently, we see from Hölder's inequality that

$$
u_{\epsilon} \cdot \nabla \theta_{\epsilon}=\nabla \cdot\left(\theta_{\epsilon} u_{\epsilon}\right) \in \text { bounded set in } L^{1}\left(0, T ; W^{-1, a}(\Omega)\right) \forall 1<a<\hat{a},
$$

where $\hat{a}=2$ if $N=2$ and $\hat{a}=6 / 5$ if $N=3$.

We also have

$$
\nabla \cdot\left(\kappa\left(\varphi_{\epsilon}, \theta_{\epsilon}\right) \nabla \theta_{\epsilon}\right) \in \text { bounded set in } L^{q}\left(0, T ; W^{-1, q}(\Omega)\right) \forall 1<q<\frac{N+2}{N+1}
$$

and

whence

$$
L^{1}(\Omega) \hookrightarrow W^{-1, a}(\Omega) \forall 1<a<\frac{N}{N-1},
$$

$$
g_{\epsilon} \in \text { bounded set in } L^{1}\left(0, T ; W^{-1, a}(\Omega)\right) \forall 1<a<\frac{N}{N-1} .
$$

Taking into account (31), (32) and (33) together, we find (30).

Furthermore, we can use proposition 4 with $h=\theta_{\epsilon}$, since we have (26). This yields:

$$
\left\{\begin{array}{l}
\varphi_{\epsilon} \in \text { bounded set in } L^{2}\left(0, T ; H_{0}^{1}(\Omega)\right) \cap L^{\infty}\left(0, T ; L^{2}(\Omega)\right), \\
\varphi_{\epsilon, t}-\theta_{\epsilon} \in \text { bounded set in } L^{\sigma}\left(0, T ; H^{-1}(\Omega)\right) .
\end{array}\right.
$$

Some consequences of these estimates are the following:

- $\varphi_{\epsilon} \in$ compact set in $L^{2}(Q)$

(because one has (34) and (29) and the embedding $H_{0}^{1}(\Omega) \hookrightarrow L^{2}(\Omega)$ is compact).

- $\theta_{\epsilon} \in$ compact set in $L^{q}\left(0, T ; L^{b}(\Omega)\right) \forall 1<q<\frac{N+2}{N+1}, 1<b<\frac{N q}{N-q}$ (because one has (28) and (30) and the embedding $W_{0}^{1, q}(\Omega) \hookrightarrow L^{b}(\Omega)$ is compact). 
- $u_{\epsilon} \in$ compact set in $L^{2}(0, T ; H)$

(because one has (25) and the embedding $V \hookrightarrow H$ is also compact).

Therefore, at least for a subsequence, we have:

$$
\varphi_{\epsilon} \rightarrow \varphi \text { weakly in } L^{2}\left(0, T ; H_{0}^{1}(\Omega)\right) \text {, strongly in } L^{2}(Q) \text { and a.e., }
$$

$\theta_{\epsilon} \rightarrow \theta$ weakly in $L^{q}\left(0, T ; W_{0}^{1, q}(\Omega)\right)$, strongly in $L^{q}\left(0, T ; L^{b}(\Omega)\right)$ and a.e.,

$$
u_{\epsilon} \rightarrow u \text { weakly in } L^{2}(0, T ; V) \text {, strongly in } L^{2}(Q)^{N} \text { and a.e., }
$$

$$
\begin{array}{r}
\varphi_{\epsilon, t} \rightarrow \varphi_{t} \text { weakly in } L^{\sigma}\left(0, T ; H^{-1}(\Omega)\right), \\
u_{\epsilon, t} \rightarrow u_{t} \text { weakly in } L^{\sigma}\left(0, T ; V^{\prime}\right) .
\end{array}
$$

for all $1<q<(N+2) /(N+1)$ and $1<b<N q /(N-q)$.

\subsection{Passage to the limit and conclusions}

The convergence properties (35)-(39) are enough to prove that we can pass to the limit in the equations and initial conditions satisfied by $u_{\epsilon}$ and $\varphi_{\epsilon}$. This is well known.

We will now show that $\theta$ solves (2) in the renormalized sense. In fact, it is just here where we have to begin to assume that $N=2$.

Since $N=2$, we have $u \in L^{2}\left(0, T ; V^{\prime}\right)$ and, therefore,

$$
\frac{1}{2}\left\|u\left(t_{2}\right)\right\|_{2, \Omega}^{2}-\frac{1}{2}\left\|u\left(t_{1}\right)\right\|_{2, \Omega}^{2}+\int_{t_{1}}^{t_{2}} \int_{\Omega} \nu(\varphi, \theta)|D(u)|^{2} d x d t=\int_{t_{1}}^{t_{2}} \int_{\Omega} f \cdot u d x d t
$$

for all $t_{1}, t_{2} \in[0, T]$.

One of the delicate points of the argument is to prove that $D\left(u_{\epsilon}\right) \rightarrow D(u)$ strongly in $L^{2}(Q)^{2 \times 2}$. To this purpose, we argue as in the proof of proposition 5 (but now letting $\epsilon \rightarrow 0^{+}$).

We first notice that

$$
\left\{\begin{array}{l}
u_{\epsilon}(T) \rightarrow u(T) \text { weakly in } H \text { and } \\
\nu\left(\varphi_{\epsilon}, \theta_{\epsilon}\right)^{1 / 2} D\left(u_{\epsilon}\right) \rightarrow \nu(\varphi, \theta)^{1 / 2} D(u) \text { weakly in } L^{2}(Q)^{2 \times 2}
\end{array}\right.
$$

Then, we multiply the regularized motion equation (23) by $u_{\epsilon}$ and we integrate over $\Omega \times(0, T)$. Using Green's formula, the fact that div $u_{\epsilon} \equiv 0$ and Hölder's and Young's inequalities, we deduce that

$$
\frac{1}{2}\left\|u_{\epsilon}(T)\right\|_{2, \Omega}^{2}+\iint_{Q} \nu\left(\varphi_{\epsilon}, \theta_{\epsilon}\right)\left|D\left(u_{\epsilon}\right)\right|^{2} d x d t=\iint_{Q} f \cdot u_{\epsilon} d x d t+\frac{1}{2}\left\|u_{0}\right\|_{2, \Omega}^{2} .
$$

From (35), we get

$$
\begin{gathered}
\lim _{\epsilon \rightarrow 0}\left[\frac{1}{2}\left\|u_{\epsilon}(T)\right\|_{2, \Omega}^{2}+\iint_{Q} \nu\left(\varphi_{\epsilon}, \theta_{\epsilon}\right)\left|D\left(u_{\epsilon}\right)\right|^{2} d x d t\right] \\
=\iint_{Q} f \cdot u d x d t+\frac{1}{2}\left\|u_{0}\right\|_{2, \Omega}^{2} .
\end{gathered}
$$


On the other hand, $u$ is a solution of (3), whence

$$
\frac{1}{2}\|u(T)\|_{2, \Omega}^{2}+\iint_{Q} \nu(\varphi, \theta)|D(u)|^{2} d x d t=\iint_{Q} f \cdot u d x d t+\frac{1}{2}\left\|u_{0}\right\|_{2, \Omega}^{2}
$$

and

$$
\begin{aligned}
\lim _{\epsilon \rightarrow 0} & {\left[\frac{1}{2}\left\|u_{\epsilon}(T)\right\|_{2, \Omega}^{2}+\iint_{Q} \nu\left(\varphi_{\epsilon}, \theta_{\epsilon}\right)\left|D\left(u_{\epsilon}\right)\right|^{2} d x d t\right] } \\
= & \frac{1}{2}\|u(T)\|_{2, \Omega}^{2}+\iint_{Q} \nu(\varphi, \theta)|D(u)|^{2} d x d t .
\end{aligned}
$$

From (40), (41) and the a.e. convergence of $\varphi_{\epsilon}$ and $\theta_{\epsilon}$, the desired strong convergence of $D\left(u_{\epsilon}\right)$ is ensured.

A consequence is that

$$
g_{\epsilon} \rightarrow \nu(\varphi, \theta) D(u): D(u) \text { strongly in } L^{1}(Q) .
$$

Now, it can be shown that $\theta_{\epsilon}$ is a Cauchy sequence in $C^{0}\left([0, T] ; L^{1}(\Omega)\right)$ and, moreover,

$$
\lim _{\epsilon \rightarrow 0^{+}} \iint_{Q}(T-t)\left|\nu\left(\varphi_{\epsilon}, \theta_{\epsilon}\right) \nabla T_{R}\left(\theta_{\epsilon}\right)-\nu(\varphi, \theta) \nabla T_{R}(\theta)\right|^{2} d x d t=0
$$

for every $R>0$. In particular, $T_{R}\left(\theta_{\epsilon}\right)$ converges strongly to $T_{R}(\theta)$ in $L^{2}\left(0, T^{\prime} ; H_{0}^{1}(\Omega)\right)$ for every $R>0$ and every $T^{\prime}<T$. All this is implied by (26), (27) and (42), but is not immediate; For more details, we refer for instance to [18, Appendix E].

This shows that there exists a subsequence, still indexed with $\epsilon$, such that we have the following for any $\beta \in W^{2, \infty}(\mathbb{R})$ such that Supp $\beta^{\prime} \subset[-R, R]$ :

$$
\begin{aligned}
& \theta_{\epsilon} \rightarrow \theta \text { and } \beta\left(\theta_{\epsilon}\right) \rightarrow \beta(\theta) \quad \text { weakly in } \quad L^{q}\left(0, T ; W_{0}^{1, q}(\Omega)\right) \cap L^{\tau}(Q) \text {, } \\
& T_{R}\left(\theta_{\epsilon}\right) \rightarrow T_{R}(\theta) \quad \text { strongly in } L^{2}\left(0, T ; H_{0}^{1}(\Omega)\right) \text {. }
\end{aligned}
$$

Furthermore, by multiplying (21) by $\beta^{\prime}\left(\theta_{\epsilon}\right)$, we also see that

$$
\beta\left(\theta_{\epsilon}\right)_{t}-k \Delta \beta\left(\theta_{\epsilon}\right)+\operatorname{div}\left(u_{\epsilon} \beta\left(\theta_{\epsilon}\right)\right)+k \beta^{\prime \prime}\left(\theta_{\epsilon}\right)\left|\nabla \theta_{\epsilon}\right|^{2}=\beta^{\prime}\left(\theta_{\epsilon}\right) g_{\epsilon} \quad \text { in } \quad Q .
$$

Let us multiply (45) by a test function $\eta \in C^{1}\left([0, T] ; H_{0}^{1}(\Omega)\right) \cap L^{\infty}(Q)$ such that $\eta(t)=0$ in a neighborhood of $T$ and let us integrate over $Q$. After some usual integrations by parts, using (22) and observing the properties of $\eta$, we get:

$$
\begin{gathered}
-\iint_{Q} \beta\left(\theta_{\epsilon}\right) \eta_{t} d x d t+\iint_{Q} \kappa\left(\varphi_{\epsilon}, \theta_{\epsilon}\right) \nabla \beta\left(\theta_{\epsilon}\right) \cdot \nabla \eta d x d t \\
+\iint_{Q} \kappa\left(\varphi_{\epsilon}, \theta_{\epsilon}\right) \nabla \theta_{\epsilon} \cdot \nabla \beta^{\prime}\left(\theta_{\epsilon}\right) \eta d x d t+\iint_{Q}\left(u_{\epsilon} \cdot \nabla \beta\left(\theta_{\epsilon}\right)\right) \eta d x d t \\
=\iint_{Q} \beta^{\prime}\left(\theta_{\epsilon}\right) g_{\epsilon} \eta d x d t+\int_{\Omega} \beta\left(\theta_{0}\right) \eta(x, 0) d x .
\end{gathered}
$$

Thanks to (42) and (43)-(44), we can take $\epsilon \rightarrow 0$ in this identity. This gives (7) for functions $\eta$ of this kind. By a standard dnsity argument, we deduce (7) for all $\eta \in C^{1}\left([0, T] ; H_{0}^{1}(\Omega)\right) \cap L^{\infty}(Q)$ with $\left.\eta\right|_{t=T}=0$.

This ends the proof of theorem 2 . 
Remark 1 The existence of weak-renormalized solutions to other related systems has been established in other papers; see for instance [?] and [2]; see also [15] for the case of a viscous, compressible and heat conducting fluid.

Remark 2 If we neglect convection and we omit the transport term $(u \cdot \nabla) u$ in the motion equation (3), the argument used in the proof of theorem 2 remains valid for $N=3$. On the other hand, the uniqueness of the weak-renormalized solution to (1)(5) is unknown even when $N=2$ and the coefficients $\nu$ and $\kappa$ are constant.

Remark 3 It is readily seen that the previous proof of theorem 2 does not work in the case $N=3$. Indeed, the strong convergence in $L^{2}(Q)^{3 \times 3}$ of the gradients of the approximate velocity fields is out of scope; in fact, this is a major difficulty even for similar approximations to the Navier-Stokes equations. Unfortunately, we do need this convergence to take limits in the equation for $\theta_{\epsilon}$ if we are looking for a weak-renormalized solution in the sense of definition 1 . Hence, in the threedimensional case, it seems appropriate to reformulate the problem, perhaps in terms of other variables; see [16] for some partial results for three-dimensional flows; see also [9], where a three-dimensional problem close to (1)-(5) with Fourier-Navier (slip) conditions on $u$ has been solved satisfactorily.

\section{References}

[1] D.M Anderson, A phase-field model of solidification with convection, Phys. D 135, 175-194.

[2] A. Attaoui, Weak-renormalized solution for a nonlinear boussinesq system, Differ. Integral Equations 22 (5-6), 465-494.

[3] C. Beckermann, Modeling melt convection in phase-field simulations of solidification, J. Comp. Phys. 154, 468.

[4] D. Blanchard, Existence and uniqueness of a renormalized solution for a fairly general class of nonlinear parabolic problems, J. Differ. Equatios 177 (2), 331374.

[5] D. Blanchard, A few result on coupled systems of thermomechanics, In "On the notions of solution to nonlinear elliptic problems: results and developments" Vol. 23, Quaderni di Matematica, 145-182.

[6] D. Blanchard, Truncations and monotonicity methods for parabolic equations, Nonlinear. Analys., Theory, Methods \& Applications 21, No. 10, 725-743.

[7] L. Boccardo, Nonlinear elliptic and parabolic equations involving measure data, J. Func. Analysis 87, 149-169.

[8] N. Bruyère, Existence et unicité de la solutions faible-renormalisé pour un système non lináire de boussinesq, C. R. Math. Acad. Sci. Paris 346, No. 9-10, 521-526. 
[9] M. Bulíček, A navier-stokes-fourier system for incompressible fluids with temperature dependent material coefficients, Nonlinear Anal. Real World Appl. 10, no. 2, 992-1015.

[10] G. Caginalp, An analysis of phase field model of a free boundary, Arch. Rat. Mech. Anal. 92, 205-245.

[11] G. Caginalp, A derivation and analysis of phase field models of thermal alloy, Annals of Phys 237, 66-107.

[12] R. J. DiPerna, On the cauchy problem for boltzmann equations:, Ann. Math. 130, 321-366.

[13] R. J. DiPerna, On the fokker-plank-boltzmann equation, Comm. Math. Phys. 120, $1-23$.

[14] L. C. Evans, Partial differential equations, vol. 19, American Mathematical Society, Paris.

[15] E. Feireisl, On the motion of a viscous, compressible, and heat conducting fluid, Indiana Univ. Math. J. 53, no. 6, 1705-1738.

[16] J. Frehse, Renormalized estimates for solutions to the navier-stokes equation, Funct. Approx. Comment. Math. 40, part 1, 11-32.

[17] J.-L. Lions, Quelques méthodes de résolution de problèmes aux limites non linéaires, Dunod, Gauthiers-Villars, Paris.

[18] P.-L. Lions, Mathematical topics in fluid mechanics:, Clarendon Press Oxford 1.

[19] M.T.G. Montesinos, Renormalized solutions to a nonlinear parabolic-elliptic system, SIAM J. Math. Anal. 36, No. 6, 1991-2003.

[20] C. Moroşanu, A generalized phase-field system, J. Math. Anal. Appl. 273, 515540.

[21] L. Nirenberg, On elliptic partial differential equation, Ann. Scuola Norm. Sup. 13, 116-162.

[22] R. Témam, Navier-stokes equations:, American Mathematical Society. 